\title{
AN EXTENSION ON THE HARDY-HILBERT INTEGRAL INEQUALITY AND ITS APPLICATIONS
}

\author{
ZHOU Yu AND GAO MingZHE
}

Abstract. In this article, it is shown that some new extensions on the Hardy-Hilbert inequality related to exponent function can be established by introducing a parameter $\lambda\left(1-\frac{q}{p}<\lambda \leqslant 2\right.$, $\frac{1}{p}+\frac{1}{q}=1$ and $\left.p \geqslant q>1\right)$ and two exponent functions $A a^{x}(A>0, a>1)$ and $B b^{y}$ $(B>0, b>1)$. In particular, for the case $p=2$, an extension of the Hilbert integral inequality is built. As an application, a new Hardy-Littlewood integral inequality is given.

Mathematics subject classification (2000): 26D15, 33B10.

Key words and phrases: Exponent function, weight function, beta function, Hardy-Hilbert inequality, Hilbert integral inequality, Hardy-Littlewood integral inequality.

\section{REFERENCES}

[1] Hardy, G. H., Littlewood, J. E., And G.Polya, Inequalities. Cambridge: Cambridge Univ. Press, 1952.

[2] Mitrinovic, D. S., PECARIC, J. E. AND FinK A. M., Inequalities involving functions and their integral and derivatives, Boston, Kluwer Academic, 1991.

[3] Gao Mingzhe And GaO XuemeI, On the generalized Hardy-Hilbert inequality and its applications, Math. Inequal. Appl., Vol. 7, 1(2004), 19-26.

[4] Yang Bicheng And L. Debnath, On the extended Hardy-Hilbert's inequality. J. Math. Anal. Appl., Vol. 272, 1(2002), 187-199.

[5] Kuang Jichang, On new extensions of Hilbert's integral inequality. J. Math. Anal.Appl., Vol. 235, 2(1999), 608-614.

[6] Mario Krnic, Gao Mingzhe, Josip Pecaric and Gao Xuemei, On the Best Constant in Hilbert's Inequality, Math. Inequal. Appl., Vol. 8, 2(2005), 317-329.

[7] PeCARIC, J. E., Generalization of inequalities of Hardy-Hilbert type. Math. Inequal. Appl., Vol. 7, 2 (2004), 217-225.

[8] Jia WeiJian, GaO Mingzhe And Gao XuemeI, On a Weighted Hardy-Hilbert's Type Inequality, International Journal of Pure and Applied Mathematics, Vol. 14, 2(2004), 255-269.

[9] Gao Mingzhe, On Hilbert's inequality and its applications, J. Math. Anal. Appl., Vol. 212, 1(1997), 316-323.

[10] Gao Mingzhe, Tan Li And L. Debnath, Some improvements on Hilbert's integral inequality, J. Math. Anal. Appl., Vol. 229, 2(1999), 682-689. 
Journal of Mathematical Inequalities www.ele-math.com jmilele-math.com 